\title{
Use of the Frailty Index and FRAIL-NH Scale for the Assessment of the Frailty Status of Elderly Individuals Admitted in a Long-term Care Hospital in Korea
}

\author{
Hyuk Ga ${ }^{1}$, Chang Won Won ${ }^{2}$, Hee-Won Jung ${ }^{3}$ \\ ${ }^{1}$ Institute of Geriatric Medicine, Incheon Eun-Hye Hospital, Incheon, ${ }^{2}$ Elderly Frailty Research Center, Department of Family Medicine, \\ College of Medicine, Kyung Hee University, Seoul, ${ }^{3}$ Graduate School of Medical Science and Engineering, Korea Advanced Institute \\ of Science and Technology (KAIST), Daejeon, Korea
}

Corresponding Author:

Hee-Won Jung, MD

Graduate School of Medical

Science and Engineering, Korea

Advanced Institute of Science and Technology (KAIST), 291,

Daehak-ro, Yuseong-gu, Daejeon 34141, Korea

Tel: $+82-42-350-4283$

Fax: +82-42-350-4269

E-mail: dr.ecsta@gmail.com

Received: November 27, 2017

Revised: December 27, 2017

Accepted: January 27, 2018

\begin{abstract}
Background: Numerous elderly individuals with multimorbidity and impaired function are admitted in long-term care hospitals (LTCHs) in Korea. In this study, we aimed to describe the frailty status of elderly patients admitted in a LTCH using the FRAIL-NH scale and to identify the clinical relevance of frailty status on clinical outcomes, including death. Methods: We retrospectively reviewed the medical records of 100 elderly patients who were hospitalized and died in an LTCH from March 2011 to February 2017. The monthly assessment results obtained from the inpatients' data set (IDS) were used as main data sources for the 6-item FRAIL-NH scale and frailty index that was composed of 22 newly established items. Results: The mean frailty index of the patients included in the analysis (mean age, 81.5 \pm 7.2 years; men, 53\%) was 0.60 (standard deviation [SD], 0.10 ; range, $0.28-0.80$ ). The distribution of the FRAIL-NH score in this population was in accordance with the 22-item frailty index, which shows a standardized beta of $0.571(p<0.001, R=0.572)$. When the patients were categorized based on the FRAIL-NH score, the mean survival durations of the more fail group (FRAIL-NH >10, $\mathrm{n}=49$ ) and less frail group (FRAIL-NH $\leq 10, n=51)$ were 529.3 days $(S D, 453.4)$ and 888 days (SD, 679.9), respectively. Similarly, the frailty index was associated with earlier mortality. Conclusion: Frailty is extremely common in elderly patients admitted in an LTCH and can be easily measured using the FRAIL-NH scale that utilizes the IDS of LTCHs in Korea. Since frailty is associated with earlier mortality, the assessment of frailty status in patients admitted in LTCHs may be helpful in clinical decision-making.
\end{abstract}

Key Words: Frail elderly, Frailty index, Long-term care, Aged care facilities

\section{INTRODUCTION}

Because of the aging population in Korea, geriatricians face an obvious problem in their practice. Although with the rapid expansion of life expectancies in older adults, the structure of traditional family caregiving has been changed, and the concept of healthy aging lagged behind, as experienced by Japan decades earlier. ${ }^{1)}$ Consequently, in Korea, several elderly individuals experience multimorbidity, ${ }^{2)}$ functional decline, and poor quality of life with insufficient social resources. ${ }^{3)}$ Furthermore, the current health care system in Korea requires the admission of elderly individuals with functional impairment in nursing homes or long-term care hospitals (LTCHs). ${ }^{4}$ In this setting, elderly individuals sometimes receive life-sustaining therapies or unnecessary treat- ments, including potentially inappropriate medications with questionable benefits on the quality of life of this population. ${ }^{5)}$

In developed countries, such as the United States, the United Kingdom, and Japan, the concept of subacute care and long-term care had fulfilled the health care needs of patients discharged from acute hospitals, facilitating them to return to the community. ${ }^{6)}$ However, the LTCH model in Korea focused on assisting elderly individuals with activities of daily living $(A D L)$, chronic nursing care, and palliative care with no specified admission or discharge criteria, giving them only little difference in function with nursing homes in general. ${ }^{4,7)}$ In addition, when the health of nursing home patients deteriorates, which results to a demand for medical treatment, such as intravenous antibiotics or mechanical ventilation, they usually end up in LTCHs. In this regard, the functional dependence of patients admitted in LTCHs in Korea 
is extremely high, as described in a previous study on this population. ${ }^{8)}$

Frailty is defined as a state of increased vulnerability to possible stressors owing to a decreased physiological reserve in elderly individuals, ${ }^{9,10)}$ and it leads to adverse outcomes in geriatric patients, including injuries due to falls, ${ }^{11)}$ worsening of sarcopenia, ${ }^{12)}$ functional declines, and even death. ${ }^{13)}$ In this context, frailty in elderly individuals admitted in LTCHs in Korea must be addressed to ensure appropriate therapeutic decision-making because the prevalence of frailty associated with disability or multimorbidity is the primary cause of admission in an LTCH. In this regard, a group suggested that frailty status can be measured using the FRAIL-NH scale that combined the parameters of existing assessment tools. ${ }^{14-16)}$ However, to date, no study on the frailty in elderly patients admitted in LTCHs in Korea has been conducted yet.

Thus, this study aimed to describe the frailty status of elderly individuals admitted in LTCHs in Korea utilizing the FRAIL-NH scale that used the nurses' monthly assessment results obtained from the inpatients' data set (IDS). In addition, we examined the clinical relevance of the frailty status of elderly individuals admitted in LTCHs on health outcomes, such as death and worsening of general conditions.

\section{MATERIALS AND METHODS}

\section{Study Population}

We obtained the records of patients admitted in a 269-bed capacity chronic care hospital located in Incheon, South Korea. This hospital is focused on supportive and medical care for physically and mentally impaired elderly patients, such as those with dementia. Because numerous elderly individuals with functional impairment and multimorbidity stayed at this hospital until their death, we collected the medical records of 100 randomly selected patients aged 65 years or older who died from March 2011 to February 2017. Three time points were used for this study: initial admission, 3 months after the initial admission, and the last evaluation before the death of patients. In this study, the Institutional Review Board of the the hospital approved the retrieval and allowed authors to review the patients' records with no need for the informed consent.

\section{FRAIL-NH Scale}

We adopted the previously published 7-item FRAIL-NH scale by Theou et al. ${ }^{16)}$ (energy, transferring, mobility, continence, weight loss, feeding, and dressing). Since the healthcare workers in LTCHs in Korea assess the ADLs and the general conditions of the patients, such as weight and cognitive status, on a monthly basis to claim specified daily fees for care service, these datasets were used to establish the 6-item FRAIL-NH scale. Because we retrospectively retrieved variables recorded in the IDS, energy cannot be used as an item of the FRAIL-NH scale. As a result, the 6-item FRAIL-NH scale was established with the following scoring scheme: (1) transferring: 0 for an unassisted transfer, 1 for needs some help or observation in transferring, and 2 for substantial or complete help in transferring; (2) mobility: 0 for patients who can get out from their room without help, 1 for needs some help or observation in getting out from their room, and 2 for substantial or complete help in getting out from their room; (3) continence: 0 for unassisted toilet use, 1 for needs some help or observation during toilet use, and 2 for substantial or complete help during toilet use; (4) weight loss within the last 3 months: 0 for no weight loss, 1 for $1-3 \mathrm{~kg}$ of weight loss, and 2 for $>3 \mathrm{~kg}$ of weight loss; (5) feeding: 0 for unassisted feeding, 1 for needs some help or observation during feeding, and 2 for substantial or complete help during feeding; and (6) dressing: 0 for getting on or off their clothes without assistance, 1 for requiring some help or observation during dressing, and 2 for substantial or complete help during dressing.

\section{Frailty Index}

With the use of the medical records, the frailty index was established using the concept of the accumulation of deficit model, ${ }^{17)}$ which consists of 22 items that may reflect possible health deficits. This model is described in Supplementary Table 1, and by dividing the total amount of deficits by 22, a frailty index ranging from 0 to 1 was calculated.

\section{Outcome Measures}

Since all the patients included in this study eventually died after chronic care in this hospital, the length of hospital stay was measured as a key outcome. In addition, a fall that occurred within the last 6 months was included, and the requirement for tube feeding before a patients' death was considered as a supplementary outcome.

\section{Other Clinical Variables}

Age upon admission, sex, residential status (home, nursing home, acute care hospital, and LTCH) before admission, and history of formal education (none, $<6$ years, 6-12 years, $>12$ years, and unknown) were measured as basic demographic variables. Moreover, baseline clinical variables, such as the number of medications that patients regularly take, presence of diabetes, hypertension, and stroke, and hemoglobin and albumin levels, were recorded. The presence of geriatric conditions, including restrictions in $A D L$, cognitive impairment based on the Korean Mini-Mental State Examination (MMSE), ${ }^{18)}$ depressive mood, delirious symptom, and dysphagia, were 
Hyuk Ga, et al.

also recorded.

\section{Statistical Analysis}

The Mann-Whitney U-test and chi-square test were used for continuous and categorical variables, respectively, to compare the baseline characteristics of the patients based on the FRAIL-NH score. Furthermore, a linear regression analysis was carried out to assess the associations between FRAIL$\mathrm{NH}$ score and various clinical variables. The Kaplan-Meier plot, log-rank test, and Cox regression were used to assess the effect of FRAIL-NH score on the length of hospital stay. The statistical analyses were performed using STATA 15.0 (StataCorp, College Station, TX, USA).

\section{RESULTS}

\section{Baseline Characteristics}

Among the 100 patients included in the analysis, 53 were men (mean age, 76.5 years; standard deviation [SD], 8.0) and 47 were women (mean age, 81.5 years; SD, 7.2; $\mathrm{p}=0.002$ ). In this population, the mean baseline FRAIL-NH score was 9.98 (SD, 2.01), which shows that 51 and 49 patients had

Table 1. Baseline characteristics of the patients in the less frail (FRAIL-NH $\leq 10$ ) and more frail (FRAIL-NH $>10$ ) groups admitted in an long-term care hospital

\begin{tabular}{|c|c|c|c|}
\hline Characteristic & Less frail $(n=51)$ & More frail $(n=49)$ & p-value \\
\hline Age (yr) & $78.4 \pm 9.0$ & $79.4 \pm 6.9$ & 0.356 \\
\hline Female sex & $24(47.1)$ & $23(46.9)$ & 0.990 \\
\hline Education history & & & 0.338 \\
\hline None & $9(17.6)$ & $9(18.4)$ & \\
\hline$<6$ Years & $9(17.6)$ & $13(26.5)$ & \\
\hline $6-12$ Years & $12(23.5)$ & $15(30.6)$ & \\
\hline$>12$ Years & $4(7.8)$ & $5(10.2)$ & \\
\hline Unknown & $17(33.3)$ & $7(14.3)$ & \\
\hline Hypertension & $21(41.2)$ & $24(49.0)$ & 0.433 \\
\hline Diabetes & $10(19.6)$ & $12(24.5)$ & 0.556 \\
\hline Stroke & $23(45.1)$ & $25(51.0)$ & 0.553 \\
\hline Number of medications & $5.5 \pm 2.4$ & $6.3 \pm 3.4$ & 0.289 \\
\hline MMSE score & $9.3 \pm 7.3$ & $6.3 \pm 6.5$ & 0.048 \\
\hline Falls within the last 6 months & $0(0)$ & $1(2.0)$ & 0.353 \\
\hline Requirement for tube feeding & $14 \pm 27.5$ & $20 \pm 40.8$ & 0.158 \\
\hline Hemoglobin level & $11.7 \pm 2.0$ & $12.3 \pm 1.9$ & 0.069 \\
\hline Serum albumin level & $3.5 \pm 0.4$ & $3.6 \pm 0.4$ & 0.474 \\
\hline
\end{tabular}

Values are presented as mean \pm standard deviation or number (\%).

MMSE, Mini-Mental State Examination.
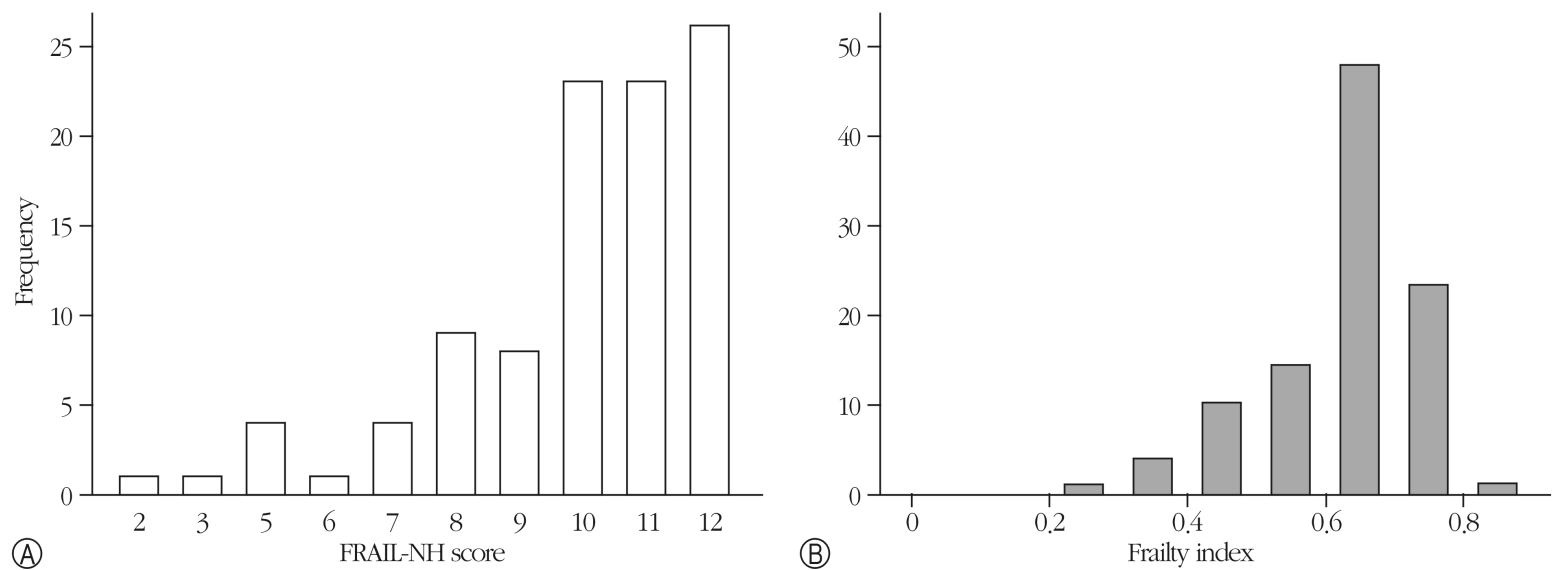

Fig. 1. Frequency distribution of the patients according to the FRAIL-NH score (A) and frailty index (B). 


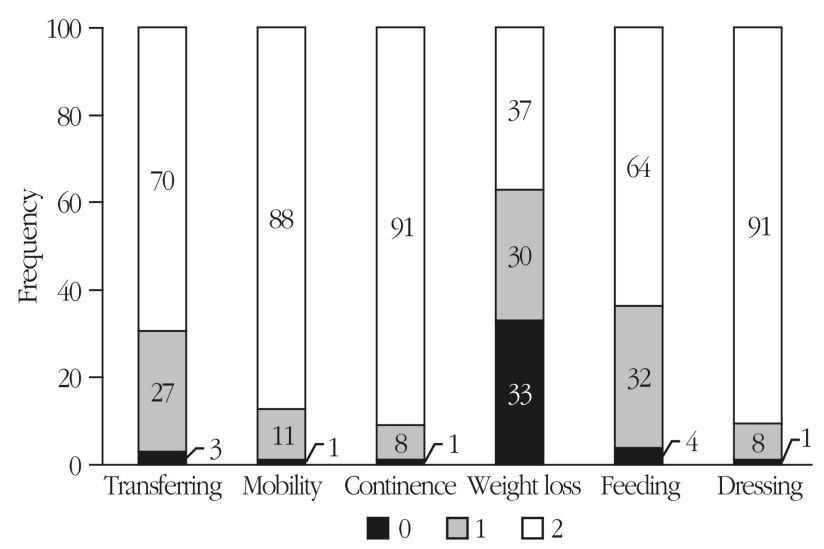

Fig. 2. Frequency of the score* of each sub item included in the FRAIL-NH scale in the study. "Scoring scheme: (1) transferring: 0 for unassisted transfer, 1 for needs some help or observation in transferring, and 2 for substantial or complete help in transferring; (2) mobility: 0 for patients who can get out from their room without help, 1 for needs some help or observation in getting out from their room, and 2 for substantial or complete help in getting out from their room; (3) continence: 0 for unassisted toilet use, 1 for needs some help or observation during toilet use, and 2 for substantial or complete help during toilet use; (4) weight loss within the last 3 months: 0 for no weight loss, 1 for 1-3 kg of weight loss, and 2 for $>3 \mathrm{~kg}$ of weight loss; (5) feeding: 0 for unassisted feeding, 1 for needs some help or observation during feeding, and 2 for substantial or complete help during feeding; (6) dressing: 0 for getting on or off their clothes without assistance, 1 for requiring some help or observation during dressing, and 2 for substantial or complete help during dressing.

a FRAIL-NH score of 10 or less and 11 or higher, respectively (Fig. 1A). In addition, the distributions of the scores for each component in the FRAIL-NH scale are shown in Fig. 2, which shows that most patients had impairments in nearly all ADL domains.

The mean frailty index of the patients included in the analysis was 0.60 (SD, 0.10 ; range, $0.28-0.80$ ). The distribution of the FRAIL-NH scores in this population was in accordance with the 22-item frailty index (Fig. 2B), which shows a standardized beta of $0.571(p<0.001, R=0.572)$.

Based on the distribution of the FRAIL-NH scores of the study population, we classified the patients into the less frail group (FRAIL-NH $\leq 10$ ) and more frail group (FRAIL-NH $>10$ ) based on a mean FRAIL-NH score of 9.98. As shown in Table 1, no significant difference was observed between the 2 groups in terms of demographic and clinical variables, except for the MMSE score that was relatively lower in the more frail group.

\section{Outcome Relevance of the FRAIL-NH Scale and Frailty Index}

A survival analysis was performed using the Kaplan-Meier

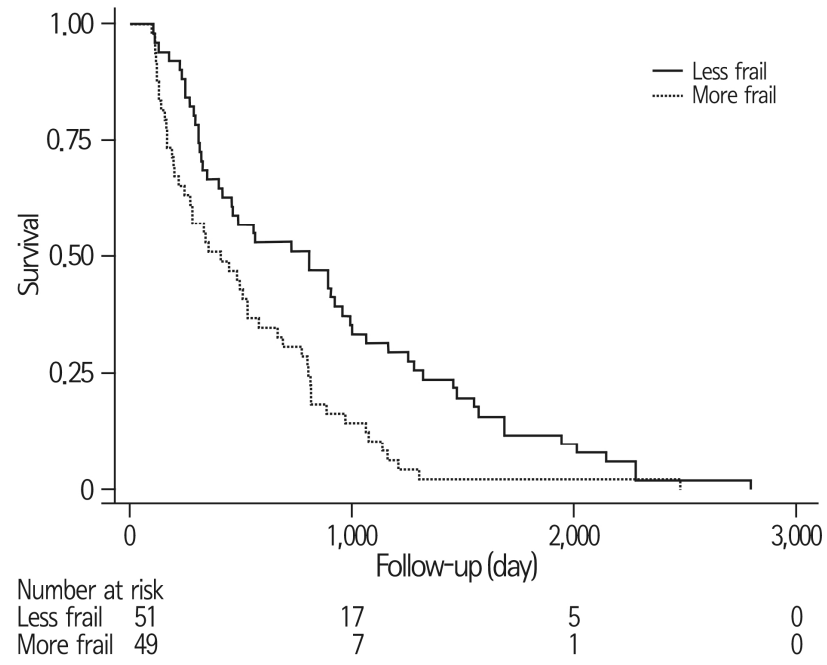

Fig. 3. Survival curve of the 100 patients in the less frail group (FRAIL-NH $\leq 10)$ and more frail group (FRAIL-NH >10). A significant difference was observed in the survival duration, with a p-value of 0.002 .

curve (Fig. 3) and log-rank test during the mean length of hospital stay, that is, 712.3 days (SD, 604.7). The mean survival durations were 888 days (SD, 679.9) and 529.3 days (SD, 453.4) in the less frail group and more frail group, respectively $(p=0.002)$. Based on the unadjusted Cox regression analysis, the hazard ratio (HR) of earlier death in the more frail group was 1.91 (95\% confidence interval [Cl], 1.27-2.88). This effect of higher FRAIL-NH score was maintained (HR, 1.29; $95 \% \mathrm{Cl}$, 1.29-2.98) even after adjusting for age, sex, and MMSE score. Similarly, a higher frailty index was associated with earlier mortality based on either unadjusted Cox regression analysis ( $\mathrm{HR}, 1.32$ [based on a 0.1 increment of the frailty index]; $95 \% \mathrm{Cl}, 1.05-1.66)$ or multivariate Cox regression analysis (HR, 1.39 [based on a 0.1 increment of frailty index]; 95\% $\mathrm{Cl}, 1.10-1.76)$ after adjusting for age, sex, and MMSE score. Within the last 6 months of hospital stay, the requirement for tube feeding was more common in the more frail group than in the less frail group based on the last assessment before death $(n=21$ in the less frail group and $n=31$ in the more frail group, $p=0.034$ ).

\section{DISCUSSION}

In this retrospective study, the frailty status of elderly patients admitted in an LTCH in Korea was evaluated using the FRAIL-NH scale that utilized data obtained from the IDS, and the clinical relevance of the frailty status in elderly individuals admitted in LTCHs on clinical outcomes was also assessed. Results showed that frailty is extremely common in patients admitted in this LTCH, with most patients who are functionally impaired at baseline. Even in these patients who are already frail at baseline, a more severe frailty accord- 
ing to the FRAIL-NH scale was associated with decreased life expectancy, and frequent tube feeding during their end of life care. To the best of our knowledge, this study first assessed the effect of frailty on the clinical outcomes in elderly individuals admitted in LTCHs in Korea.

According to a systematic review, frailty is an extremely common geriatric syndrome in nursing homes, of which a prevalence of $19.0 \%-75.6 \%$ was observed. ${ }^{19)}$ In the present study, ${ }^{19)}$ results showed that there is a substantial fraction of the elderly population with a prefrailty state $(40.1 \%$ in the pooled analysis), even though they are admitted in nursing homes. In contrast, most of the patients were already frail prior to analysis, and a small proportion of elderly individuals with a prefrailty state was observed. This result might be explained by the fact that patients with both functional restrictions on $A D L$ that require assistive care services and acute or chronic medical conditions that cannot be managed in nursing homes are admitted in LTCHs in Korea. Based on these results, there may be some elderly individuals from the underserved prefrail population with unmet needs for rehabilitation, particularly after acute admissions. By filling this gap, health workers in LTCHs can appropriately meet the healthcare needs of the patients according to their primary goal, thus improving the subacute care and rehabilitation of elderly individuals in Korea.

The FRAIL-NH scale was associated with earlier mortality in these patients who are already fail at baseline. This finding is in accordance with that of previous studies that included another study group. ${ }^{15,16)}$ In this regard, the identification of frailty status is still important for healthcare workers in determining the therapeutic treatments for elderly individuals and their eligibility for advance directives in LTCHs in Korea. For example, therapeutic targets of chronic diseases such as diabetes, hypertension, and atrial fibrillation can be further relaxed in the most frail subgroup of patients. Similarly, lifesustaining therapies, including parenteral nutrition, tube feeding, and mechanical ventilation with tracheostomy or endotracheal intubation, might be minimized with a timely discussion of the physician's order for life-sustaining treatments based on frailty status.

The present study showed a significantly high prevalence of frailty based on either the FRAIL-NH scale or frailty index in patients admitted in an LTCH. Thus, this result might be used as a basis for further studies on the frailty of elderly individuals admitted in LTCHs or nursing homes in Korea. However, this study has several limitations. First, the study retrospectively used patients' data from a single LTCH. While the hospital in the present study focuses on patients with severe disability and dementia, the patients' characteristics might vary in different hospitals. In addition, the fact that we retrieved the records of patients who already died may have caused selection bias of more frail patients, and re- stricted to address meaningful causal relationships between frail status and earlier mortality. Furthermore, the FRAIL-NH scale used in this study included 6 items, unlike the original 7-item version by Theou et al. ${ }^{16)}$ that included energy, because only the data obtained from the retrospective IDS of the patients were utilized. Therefore, further prospective, multicenter studies on frailty in elderly individuals admitted in LTCHs must be conducted to overcome the limitations of the current study.

In conclusion, frailty is an extremely common problem among elderly individuals and can be easily measured with the FRAIL-NH scale, which used data obtained from the IDS of LTCHs in Korea. Furthermore, frailty is associated with earlier mortality in patients admitted in an LTCH. With the rapidly growing aging population, more studies must be conducted to address frailty in the elderly population receiving chronic long-term care service in Korea.

Conflicts of Interest Disclosures: The researchers claim no conflicts of interest.

\section{Acknowledgments}

Dr. Hee-Won Jung was supported by the Global PhD Fellowship Program through the National Research Foundation of Korea funded by the Ministry of Education (NRF-2015H1A2A 1030117) and the Research Fund of the Korean Geriatrics Society of 2016.

\section{Supplementary Material}

Supplementary Table. 1 can be found via http://www.e-agmr. org/src/sm/agmr.2018.22.1.20.

\section{REFERENCES}

1. Tamiya N, Noguchi H, Nishi A, Reich MR, Ikegami N, Hashimoto $\mathrm{H}$, et al. Population ageing and wellbeing: lessons from Japan's long-term care insurance policy. Lancet 2011;378: 1183-92.

2. Jung HW, Kim KI. Multimorbidity in older adults. J Korean Geriatr Soc 2014;18:65-71.

3. Jung HW, Jang IY, Lee YS, Lee CK, Cho EI, Kang WY, et al. Prevalence of frailty and aging-related health conditions in older Koreans in rural communities: a cross-sectional analysis of the aging study of pyeongchang rural area. J Korean Med Sci 2016; 31:345-52.

4. Ga H, Won CW. Perspective on long term care hospitals in Korea. J Am Med Dir Assoc 2013;14:770-2.

5. Lim YJ, Kim HY, Choi J, Lee JS, Ahn AL, Oh EJ, et al. Potentially inappropriate medications by beers criteria in older outpatients: prevalence and risk factors. Korean J Fam Med 2016;37:329-33.

6. Song H. Long-term care hospital systems in developed countries and the implications for Korea. J Korean Geriatr Soc 2012;16: 
114-20.

7. Kim YB. A study on evaluation and improvement of long-term care hospitals for changing long-term care hospital fee system. Korean J Health Serv Manage 2011;5:105-17.

8. Ga H, Won CW, Lee RJ, Han IW, Kwon IS, Park BJ. Factors associated with a decline in activities of daily living in patients with dementia at geriatric hospitals: a 6 month prospective study. J Korean Geriatr Soc 2011;15:128-34.

9. Clegg A, Young J, Iliffe S, Rikkert MO, Rockwood K. Frailty in elderly people. Lancet 2013;381:752-62.

10. Cesari M. Physical frailty and sarcopenia: development of a framework for supporting interventions against incident mobility disability. Ann Geriatr Med Res 2017;21:42-8.

11. Fried LP, Tangen CM, Walston J, Newman AB, Hirsch C, Gottdiener J, et al. Frailty in older adults: evidence for a phenotype. J Gerontol A Biol Sci Med Sci 2001;56:M146-56.

12. Jung HW, Kim SW, Lim JY, Kim KW, Jang HC, Kim CH, et al. Frailty status can predict further lean body mass decline in older adults. J Am Geriatr Soc 2014;62:2110-7.

13. Jung HW, Kim SW, Ahn S, Lim JY, Han JW, Kim TH, et al. Prevalence and outcomes of frailty in Korean elderly population: comparisons of a multidimensional frailty index with two phenotype models. PLoS One 2014;9:e87958.

14. Kaehr E, Visvanathan R, Malmstrom TK, Morley JE. Frailty in nursing homes: the FRAIL-NH Scale. J Am Med Dir Assoc 2015;16:87-9.

15. Kaehr EW, Pape LC, Malmstrom TK, Morley JE. FRAIL-NH predicts outcomes in long term care. J Nutr Health Aging 2016; 20:192-8.

16. Theou O, Tan EC, Bell JS, Emery T, Robson L, Morley JE, et al. Frailty levels in residential aged care facilities measured using the frailty index and FRAIL-NH scale. J Am Geriatr Soc 2016;64:e207-12.

17. Searle SD, Mitnitski A, Gahbauer EA, Gill TM, Rockwood K. A standard procedurefor creating a frailty index. BMC Geriatr 2008;8:24.

18. Kang Y, Na DL, Hahn S. A validity study on the Korean MiniMental State Examination (K-MMSE) in dementia patients. J Korean Neurol Assoc 1997;15:300-8.

19. Kojima G. Prevalence of frailty in nursing homes: a systematic review and meta-analysis. J Am Med Dir Assoc 2015;16:940-5. 
Supplementary Table 1 . Items included in the frailty index

\begin{tabular}{|c|c|}
\hline Item & Scoring \\
\hline Dressing & $\begin{array}{l}0 \text { for dress without assistance, } 0.5 \text { for requiring some help or observation, } 1 \text { for substantial } \\
\text { or complete help }\end{array}$ \\
\hline Facial washing & $\begin{array}{l}0 \text { for washing without assistance, } 0.5 \text { for requiring some help or observation, } 1 \text { for substantial } \\
\text { or complete help }\end{array}$ \\
\hline Mouth washing & $\begin{array}{l}0 \text { for washing without assistance, } 0.5 \text { for requiring some help or observation, } 1 \text { for substantial } \\
\text { or complete help }\end{array}$ \\
\hline Body washing & $\begin{array}{l}0 \text { for washing without assistance, } 0.5 \text { for requiring some help or observation, } 1 \text { for substantial } \\
\text { or complete help }\end{array}$ \\
\hline Eating & $\begin{array}{l}0 \text { for eating without assistance, } 0.5 \text { for requiring some help or observation, } 1 \text { for substantial } \\
\text { or complete help }\end{array}$ \\
\hline Positioning & $\begin{array}{l}0 \text { for positioning without assistance, } 0.5 \text { for requiring some help or observation, } 1 \text { for } \\
\text { substantial or complete help }\end{array}$ \\
\hline Sitting & $\begin{array}{l}0 \text { for sitting up without assistance, } 0.5 \text { for requiring some help or observation, } 1 \text { for substantial } \\
\text { or complete help }\end{array}$ \\
\hline Moving & $\begin{array}{l}0 \text { for moving in the room without assistance, } 0.5 \text { for requiring some help or observation, } 1 \\
\text { for substantial or complete help }\end{array}$ \\
\hline Ambulation & $\begin{array}{l}0 \text { for ambulating outside the room without assistance, } 0.5 \text { for requiring some help or obser- } \\
\text { vation, } 1 \text { for substantial or complete help }\end{array}$ \\
\hline Toileting & $\begin{array}{l}0 \text { for use of toilet without assistance, } 0.5 \text { for requiring some help or observation, } 1 \text { for } \\
\text { substantial or complete help }\end{array}$ \\
\hline MMSE score & $0(25-), 0.25$ (21-24), $0.5(18-20), 0.75(11-17), 1(-10)$ \\
\hline Depressive mood & 1 if the patient had depressive mood \\
\hline Systolic blood pressure & 1 if $<90 \mathrm{mmHg}$ \\
\hline Diastolic blood pressure & 1 if $<60 \mathrm{mmHg}$ \\
\hline Total cholesterol & 1 if $<100 \mathrm{mg} / \mathrm{dL}$ \\
\hline Serum albumin & 1 if $<4 \mathrm{~g} / \mathrm{dL}$ \\
\hline Hemoglobin & 1 if $<12 \mathrm{~g} / \mathrm{dL}$ \\
\hline Polypharmacy & 1 if number of medications $>5$ \\
\hline Weight loss within the last 3 months & 0 for no weight loss, 0.5 for $1-3 \mathrm{~kg}$ of weight loss, 1 for $>3 \mathrm{~kg}$ of weight loss \\
\hline Comorbidity: diabetes & 1 if presence or history of the comorbidity \\
\hline Comorbidity: hypertension & 1 if presence or history of the comorbidity \\
\hline Comorbidity: stroke & 1 if presence or history of the comorbidity \\
\hline
\end{tabular}

MMSE, Mini-Mental State Examination. 